\title{
PAPER \\ New Stochastic Algorithm for Optimization of Both Side Lobes and Grating Lobes in Large Antenna Arrays for MPT
}

\author{
Naoki SHINOHARA $^{\dagger \text { a) }}$, Blagovest SHISHKOV ${ }^{\dagger \dagger}$, Hiroshi MATSUMOTO ${ }^{\dagger \dagger \dagger}$, Kozo HASHIMOTO $^{\dagger}$, Members , \\ and A.K.M. BAKI ${ }^{\dagger}$, Student Member
}

\begin{abstract}
SUMMARY The concept of placing enormous Solar Power Satellite (SPS) systems in space represents one of a handful of new technological options that might provide large scale, environmentally clean base load power to terrestrial markets. Recent advances in space exploration have shown a great need for antennas with high resolution, high gain and low side lobe level (SLL). The last characteristic is of paramount importance especially for the Microwave Power Transmission (MPT) in order to achieve higher transmitting efficiency (TE) and higher beam collection efficiency (BCE). In order to achieve low side lobe levels, statistical methods play an important role. Various interesting properties of a large antenna arrays with randomly, uniformly and combined spacing of elements have been studied, especially the relationship between the required number of elements and their appropriate spacing from one viewpoint and the desired SLL, the aperture dimension, the beamwidth and TE from the other. We propose a new unified approach in searching for reducing SLL by exploiting the interaction of deterministic and stochastic workspaces of proposed algorithms. Our models indicate the side lobe levels in a large area around the main beam and strongly reduce SLL in the entire visible range. A new concept of designing a large antenna array system is proposed. Our theoretic study and simulation results clarify how to deal with the problems of side lobes in designing a large antenna array, which seems to be an important step toward the realization of future SPS/MPT systems.

key words: microwave power transmission, large antenna array, uniform spacing, random spacing, spatial and amplitude tapering, side lobe level, grating lobes, workspace, transmitting efficiency, beam collection efficiency
\end{abstract}

\section{Introduction}

In the 1980s and 1990s in Japan, we have performed large investigations on Solar Power Satellites (SPS) and wireless power transmission via microwave (MPT), related by the abbreviations MINIX, ISY-METS, MILAX, SPORTS and SPIRITZ [1]. In such way, the center of MPT technologies research shifted from USA to Japan. The SPS is a huge solar power station in space, which transmits electric power via microwave. The SPS requires a huge phased array antenna with low side lobe levels (SLL) in order to achieve higher transmitting efficiency (TE) and higher beam collection efficiency (BCE).

In his fundamental paper [2] T. Taylor studied the

\footnotetext{
Manuscript received November 20, 2006.

Manuscript revised June 11, 2007.

${ }^{\dagger}$ The authors are with the Research Institute for Sustainable Humanosphere, Kyoto University, Uji-shi, 611-0011 Japan.

${ }^{\dagger}$ The author is with the Institute of Mathematics and Informatics Bulgarian Academy of Sciences, Sofia, Bulgaria.

${ }^{\dagger \dagger}$ The author is with Kyoto University, Kyoto-shi, 606-8501

a)E-mail: shino@ rish.kyoto-u.ac.jp

DOI: $10.1093 /$ ietcom/e91-b.1.286
} Japan. mathematical relationships involved in the radiation calculation of the line source from the point of view of function theory. Antenna design technique permits the other - than - uniform distributions of the field and in [2] has been investigated how to choose this distribution function to give a radiation pattern with prescribed properties such as, for example, narrow beamwidth of the main lobe and low side lobes. In this original paper was documented the relationships between aperture edge behavior, far side lobs, and array pattern zero locations. His analysis and insights led to a most practical technique for the synthesis of low-sidelobe beams.

A number of applications require a narrow scanned beam, but not commensurably high antenna gain. Since the array beamwidth is related to the largest dimension of the aperture, it is possible to remove many of the elements (or to "thin" an array) without significantly changing its beamwidth. For small or moderate arrays, it can be convenient to formulate the thinning procedure as a side lobe minimization problem (see [3]-[6]). [3] proposes a method that can reduce both the quantities of elements required for a given size aperture and the number of different types of transmitters which would be necessary in an array using an illumination taper. In [4] a linear array with general arbitrarily distributed elements is discussed. A matrix relationship is found between the elements of the array and its farzone pattern. In [5] general analytical expressions are presented for unequally spaced arrays. These relations allow for the analysis of the non-uniformly spaced array in terms of its equivalent uniformly spaced array. Some equivalence is made between the amplitude and spatial variation in the uniformly and non-uniformly spaced array. An array with monotonically increasing inter element spacing is presented as an example of the theory. In [6] a perturbation procedure for reducing the SL level of discrete linear arrays with uniform amplitude excitation by using non-uniform element spacing is presented. All these procedures [3]-[6] do control both peak and average SLL, but are numerically difficult to implement for large antenna arrays.

The variety of statistical procedures for array thinning exert direct control primarily on the average SLL and can produce peak side lobes for larger than the average level. A paper by Steinberg [7] compares the peak side lobes of 70 algorithmically designed aperiodic arrays with those of 170 random arrays. Both distributions are found to be nearly log-normal. The study shows that most techniques lead to 
very similar average levels.

The statistical method of Skolnik et al. [8] is straightforward to implement for large antenna arrays. The probability density of elements located within the aperture is made proportional to the amplitude of the aperture illumination of a conventional "filled" array. The selection of the element locations is performed statistically by choosing element weights as unity or zero with probabilities proportional to the filled-array taper. This procedure is readily applicable to the design of large antenna arrays. However it is not optimum and in that it does not ensure that peak side lobes are maintained bellow a given level. In [9] a thinning method is presented to realize the desired aperture distribution in a planar array antenna with elements fixed on an array lattice. In this method elements to be excited are determined by quantizing cumulative weights which are calculated from the desired aperture distribution. In [10] one investigates the use of statistical thinning and quantized element weights to produce low side lobe pattern using large circular array apertures. The last two papers develop further Skolnik et al. method [8] by using discrete, quantized output power levels for the array instead of a continuous taper.

In [11] the effects on radiation pattern when $N$ pairs of symmetrically located radiators are removed at random from a large $(2 \mathrm{No}+1)$-element linear array are studied as a statistical problem. The general analysis is valid for arrays with an arbitrary amplitude distribution which is symmetrical with respect to the center element.

The series of papers of Y. Lo [12]-[16] and [17] and especially the pioneering and fundamental paper [13] present probabilistic study of thinned antenna arrays. Lo addresses the peak-sidelobe issue and shows that a statistical description of these side lobes is possible and yields useful bounds for array design. In [17] mutual coupling effects in phased arrays with randomly located elements are studied.

Taking into account this background we can draw the following conclusion:

1. For conventionally designed arrays where all elements are spaced uniformly, there exists an upper limit to the spacing if the grating lobes are not permitted to appear in the visible region.

2. Deterministic non-uniformly spaced algorithms are numerically difficult to implement for large antenna arrays.

3. Randomly spaced algorithms (the concept of "thin" arrays) are easier to implement, but need of further study in order to determine their merits and drawbacks.

In this paper we develop further the existing algorithms and our previous research [18]-[21] and [22] proposing a new techniques to deal with side lobes and grating lobes.

\section{Randomly and Uniformly Spaced Arrays}

Consider a linear array along the $X$ axis in Cartesian coordinate system and suppose we are given $N+1$ equally excited antenna elements by isotropic radiation to be placed at random within an aperture defined by $|X| \leq a / 2$ in wavelength, in accordance with a common probability density function (pdf) $f(x)$.

Assume that the random positions $\left\{X_{n}\right\}$ are independent. Then for each sample vector $\left\{X_{n}\right\}, X_{n} \in R^{N+1}$, there is a sample radiation pattern function given by the magnitude of [13]

$$
\begin{aligned}
& P(u)=\frac{1}{N+1} \sum_{n=-N / 2}^{N / 2} \exp \left(j u x_{n}\right) \\
& P(\theta)=\frac{1}{N+1} \sum_{n=-N / 2}^{N / 2} \exp \{j 2 \pi(\sin \theta-\sin \alpha)\} X_{n}
\end{aligned}
$$

$\theta$ - the observation angle measured from the normal to the array axis

$\alpha$ - the scan angle measured from the normal to the array axis

$u=a \pi(\sin \theta-\sin \alpha)$ - the observation angle parameter

$\left\{x_{n}=2 X_{n} / a\right\}$ - normalized workspace

$a=N d_{x}$ - the aperture, measured in wavelength

We can determine the array factor $(\mathrm{AF})=|P(u)|^{2}$ or $|P(\theta)|^{2}$ as random functions. In (1) if $\left\{X_{n}\right\}$ is considered as positions of conventional uniform spacing $\left\{X_{n}=n d_{x}\right\}$ the model is automatically transformed in the deterministic one- see (2).

$$
\begin{aligned}
P(\theta) & =\frac{1}{N+1} \sum_{n=-N / 2}^{N / 2} \exp \{j 2 \pi(\sin \theta-\sin \alpha)\} n d_{x} \\
& =\frac{1}{N+1} \sum_{n=-N / 2}^{N / 2} \exp \left(j \psi_{n}\right)
\end{aligned}
$$

where $\psi_{n}=\frac{2 \pi}{\lambda}(\sin \theta-\sin \alpha) n d_{x}$ and normalized work space equals to $x_{n}=2 n d_{x} / N$. We can determine the array factor $\mathrm{AF}=|P(\theta)|^{2}$ as periodic function [23], [24].

A linear array with its peak at $\alpha$ can also have other peak values subject to the choice of spacing $d_{x}$. This ambiguity is apparent, since the summation also has a peak whenever the exponent is some multiple of $2 \pi$ or

$$
\frac{2 \pi}{\lambda}(\sin \theta-\sin \alpha) d_{x}=2 \pi p, \quad p= \pm(1,2, \ldots)
$$

Such peaks are called grating lobes and are shown from the above to occur at angles $\theta_{p}$ such that

$$
\sin \theta_{p}=\sin \alpha+\frac{p \lambda}{d_{x}}, \quad p= \pm(1,2, \ldots)
$$

for values of $p$ that define an angle with a real sine $\left(\left|\sin \theta_{p}\right| \leq 1\right)$. This implies that the maximum element spacing for an array scanned to a given angle $\alpha$ at frequency $f$ is $\lambda / 2$.

The model (1) is pure stochastic model and was first investigated by Y.T. Lo [13] and will be coded as RA (random array). The model (2) is well known deterministic model of uniform spacing and will be coded as UA (uniform array). Let's summarize the Lo's result [13], [23], who was 


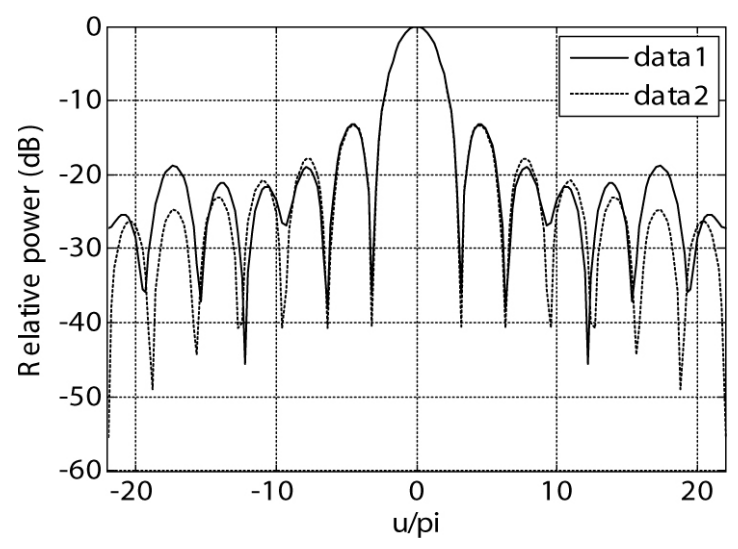

Fig. 1 Radiation characteristics of RA (data1) and UA (data2) with uniform excitation, $N=1000$, average spacing $d_{\mathrm{av}}=2 \lambda, \theta \in\left[-0.2^{\circ}, 0.2^{\circ}\right]$.

found the distribution of maximum of SLL outside of the main beam region and that at any " $u$ " the probability of antenna response being less than any level $r$ is given by

$$
\begin{aligned}
& \operatorname{Pr}\{|P(u)|<r, \text { all } u: \delta<|u|<2 \pi a\} \\
& \quad \approx\left[1-\exp ^{-N r^{2}}\right]^{[4 a]}
\end{aligned}
$$

where $\delta$ is the first positive zero of characteristic function

$$
\varphi(u)=E\{\exp (j u x)\}=\int_{-\infty}^{\infty} f(x) \exp (j u x) d x
$$

and the bracket $[4 a]$ is the integer part of $4 a$. This is a chisquared distribution with two degrees of freedom.

This expression gives the number of elements required to achieve the desired SLL (maximum, not average) with predetermined confident probability of success such as 0.9 , 0.95 , etc.

Equation (6) is important. It is seen that the mean pattern is identical to the pattern which would be obtained by taking $f(x)$ as a continuous aperture excitation.

Lo shows [23] that outside of the main beam region, the variances of the real and imaginary parts of the pattern are equal and approximately given by $1 / 2 N$, independent of the probability density function $f(x)$. This significant conclusion implies that although the pattern behavior in the main beam region is determined by $f(x)$, outside of the main beam area the variances are determined only by $N$, the number of elements, not the probability density function $f(x)$. Therefore, in many cases (unless the near-in SLL is of interest), the uniform pdf for $f(x)$ may have advantage to us to maintain a narrow beam. Keeping these suggestions in mind we will search solution of our problem in the strategy of uniform $\mathrm{pdf}$ and different non uniform excitation (illumination) functions.

Let's compare this stochastic model RA with the deterministic model of conventional uniform spacing UA model in the near side lobe region - Fig. 1 and in the entire visible range-Fig. 2.

One can make some important conclusions. In the entire visible range there is no grating lobe for RA. However,

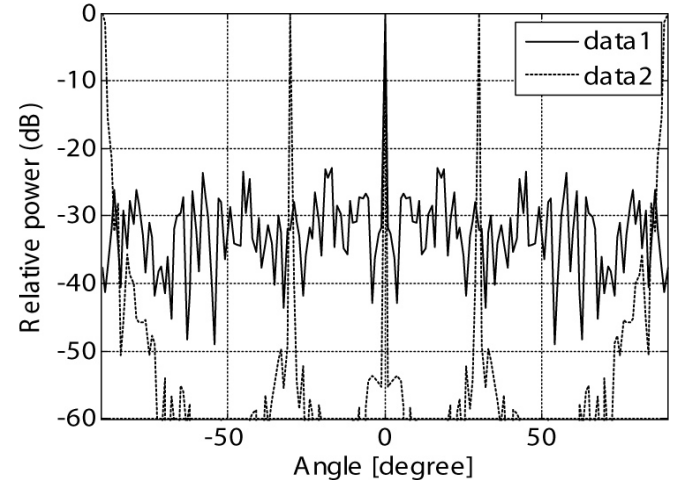

Fig. 2 Radiation characteristics of RA (data1) and UA (data2) with uniform excitation, $N=1000$, average spacing $d_{\mathrm{av}}=2 \lambda, \theta \in\left[-90^{\circ}, 90^{\circ}\right]$.

the SLL is sufficiently high for random spacing of elements in comparison with uniform spacing - UA model. When number of elements increases SLL decreases but remains still high for MPT as will be seen further. The SLL of the MPT must be decreased in order to suppress interferences to existent wireless systems. There is an estimation of interference from the MPT in the SPS to the existent wireless systems [25]. However, at the present moment, we can not decide necessary SLL of the MPT to coexistence with the existent wireless systems because conditions of the MPT and the existent wireless systems will be changed.

Second, From Fig. 1 we can see that the near SLL around the main beam is also sufficiently high for both RA and UA models. Therefore suitable excitation function will be sought to mitigate this problem.

All this has stimulated us to search for better solutions.

\section{Combined Stochastic Algorithm}

Let's consider the sample radiation pattern function given by the magnitude

$$
P(\theta)=\frac{1}{N+1} \sum_{n=-N / 2}^{N / 2} \exp \left\{j 2 \pi(\sin \theta-\sin \alpha)\left(n d_{x}+X_{n}\right)\right\}
$$

where the positions are a linear combination of deterministic one and random one. The $\mathrm{AF}|P(\theta)|^{2}$ consists of two parts. The first part is periodic function and if $d_{x}>\lambda / 2$ grating lobes can not be avoided - see Fig. 2.

Unlike, periodicity of the second part is strongly destroyed from random positions of $x_{n}$. It is equivalent of nonuniform spacing and there is no grating lobes, but on the price of relatively high SLL - see again Fig. 2.

The basic role of the algorithm for optimization both side lobes and grating lobes play positions $\left\{X_{n}\right\}$ of antenna elements. This vector $\left\{X_{n}\right\}, X_{n} \in R^{N+1}$, or its normalized version $\left\{x_{n}\right\}$ creates the work space which plays a fundamental role. Generally $\left\{x_{n}\right\}=\left\{x_{n \text { det }}\right\}+\left\{x_{n \text { rand }}\right\}$. In Sect. 2, we have considered the models $\left\{x_{n \text { rand }}\right\}$ and $\left\{x_{n \text { det }}\right\}$ separately that were called RA and UA respectively. In terms of MATLAB 


$$
\begin{aligned}
& \left\{x_{n \text { det }}=((-N / 2): 1:(N / 2)) / N\right\} \\
& \left\{x_{n \text { rand }}=\mathrm{rn}=-1 / 2+\operatorname{rand}(1, N)\right\}
\end{aligned}
$$

and each of the two workspaces operates over the interval $[-1 / 2,1 / 2]$. Equation (8) means a uniform spacing with $N$ elements in $[-1 / 2,1 / 2]$. Equation (9) means a random spacing with $N$ elements in $[-1 / 2,1 / 2]$.

Let's consider the model (7) over

$$
\left\{x_{n}\right\}=\left\{x_{n \text { det }}\right\}+\left\{x_{n \text { rand }}\right\},
$$

which operates over two times larger workspace. It is nonuniform spacing stochastic algorithm, which suppresses grating lobes, but with respect to SLL it is almost the same as RA algorithm, even with two time larger aperture.

Let's put forward the model (7) over

$$
\begin{aligned}
& \left\{x_{n}\right\}=\left\{x_{n \text { det }}\right\}+\left\{\varepsilon_{n \text { rand }}\right\} \\
& \left\{\varepsilon_{n \text { rand }}\right\}=\left\{x_{n \text { rand }}\right\} / N
\end{aligned}
$$

where $\left\{\varepsilon_{n \text { rand }}\right\}$ is a small random perturbation of the deterministic workspace and $N$ is the number of elements. This model will be called Combined Stochastic Algorithm (CSA) and will compete with existing UA and RA algorithms. Our algorithm is a non-uniform spacing stochastic algorithm, where random workspace is strongly reduced, and positions $\left\{x_{n \text { rand }}\right\} / N$ play the role of very small random perturbations around the deterministic positions $\left\{x_{n \text { det }}\right\}$. The aperture of antenna array remains unchanged. This algorithm as we will see has any advantages with respect to both UA and RA algorithms. To compare all these models with respect to near and far SLL is more convenient to represent array factor

$$
A F=|P(\theta)|^{2}
$$

or its averaged version as a function of observation angle $\theta$. So we can directly observe, that when $N$ increases the beamwidth becomes narrower and the effect of broadening the beamwidth, when we apply non-uniform amplitude tapering.

In the figures to be followed one depicts array factor $|P(\theta)|^{2}$ or its averaged version into the near zone around the main beam or entire visible range when changing any parameters. All the figures are prepared for $f=5.8 \mathrm{GHz}$ and 181 observation points $\left(N_{\text {data }}=181\right)$ for which the array factor $|P(\theta)|^{2}$ is calculated. The scan angle $\alpha$ is adjusted to zero degree. The averaged version of the AF is obtained by repeating algorithm one hundred times or more and taking the mean operator of gathered statistics. We have used averaging only to calculate area under the array factor but our CSA does not work by averaging. The final design of antenna element positions is subject to Monte Carlo simulations. However, we do not discuss the Monte Carlo simulations in this paper. All figures are prepared with uniform probability density function and with or without non-uniform amplitude excitation function.

We compare two stochastic algorithms (RA, CSA) with uniform array algorithm (UA) and must clearly distinguish uniform spacing (deterministic) from non-uniform spacing

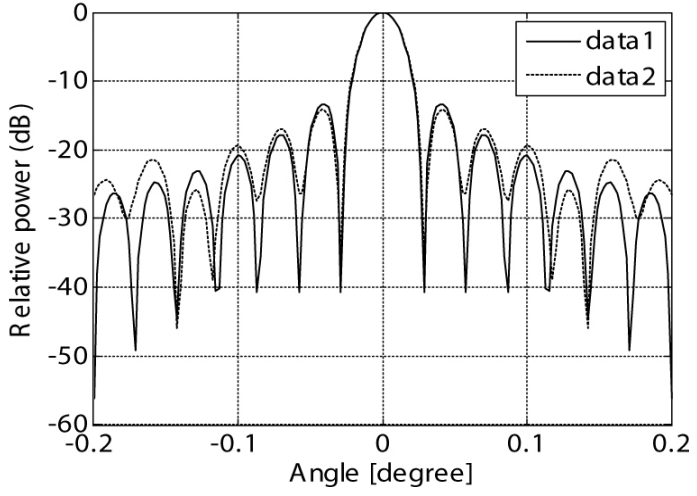

Fig. 3 Radiation characteristics of CSA (data1) and RA (data2), $N=1000$ and uniform excitation $d_{\mathrm{av}}=2 \lambda, \theta \in\left[-0.2^{\circ}, 0.2^{\circ}\right]$.

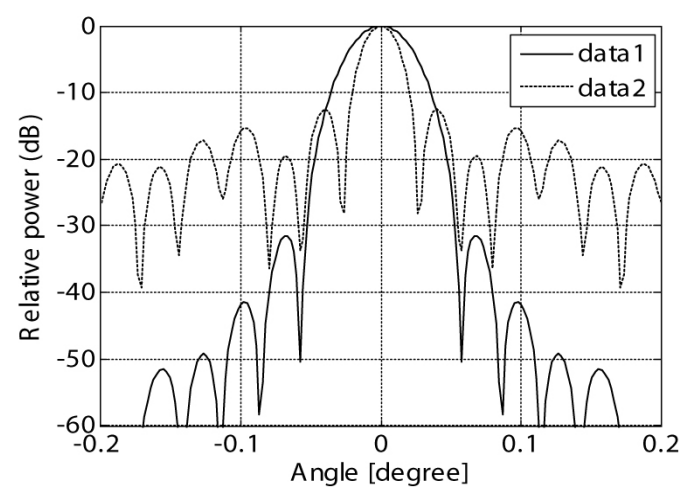

Fig. 4 Radiation characteristics of CSA (data1) and RA (data2), $N=1000$ and $\cos ^{2}(\pi x / 2)$ excitation $d_{\mathrm{av}}=2 \lambda, \theta \in\left[-0.2^{\circ}, 0.2^{\circ}\right]$.

with uniform pdf $f(x)$ (random).

In Fig. 3, one can see a comparison between the array factors of the our algorithm CSA with those of Lo's algorithm RA in the near zone of the main beam, $\theta \in$ $\left[-0.2^{\circ}, 0.2^{\circ}\right], N=1000$ and without amplitude tapering of element's excitation, or all elements (with isotropic radiation) are equally excited. There is no substantial difference between the two algorithms. The behavior of our model is almost the same as UA model.

Now let's repeat Fig. 3 by applying a non-uniform excitation function of the kind $\cos ^{2}(\pi x / 2)$. In Fig. 4, one can see that in the near zone our algorithm CSA, with non-uniform amplitude tapering, outperforms the Lo's algorithm RA and broadening of beamwidth appears [2]. Its behavior is again nearly the same as UA algorithm.

A special attention deserves the algorithm CSA into the zone larger than that around the main beam $\theta \in\left[-15^{\circ}, 15^{\circ}\right]$, see Fig. 5 and Fig. 6. For the SPS in geostationary orbit above $36,000 \mathrm{~km}$, it is very important to suppress the SLL within $\pm 8^{\circ}$ because it covers all the surface of the Earth from the geostationary orbit. One can see a strong reduction of SLL in this zone, which further is constant up to the bounds of entire visible range. The effect of the CSA is occurred by the mixing of UA and RA (see Eq. (11)). In near mainlobe, the strong reduction of the sidelobe levels is occurred 


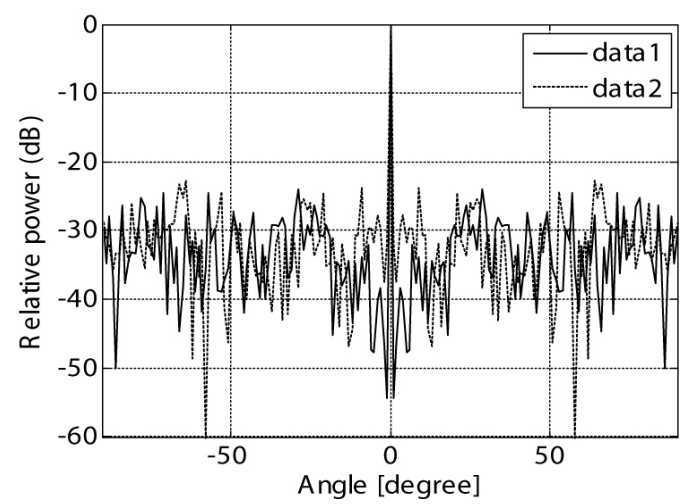

Fig. 5 Radiation characteristics of CSA (data1) and RA (data2), $N=1000$ and uniform excitation $d_{\mathrm{av}}=2 \lambda, \theta \in\left[-90^{\circ}, 90^{\circ}\right]$.

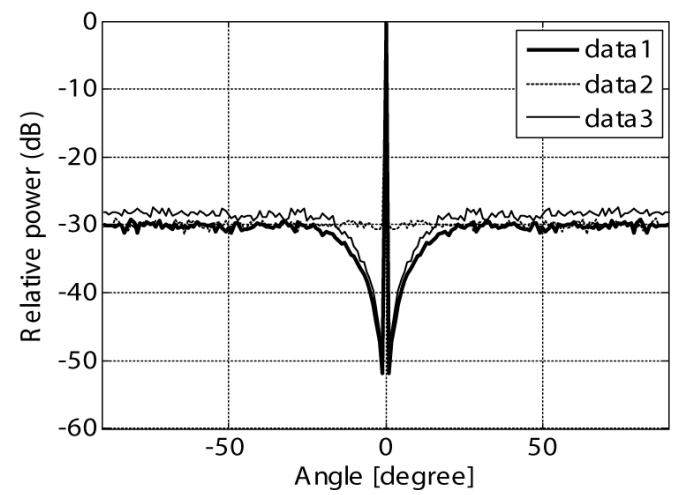

Fig. 6 Average patterns of the CSA (data1) and RA (data2) for uniform excitation and CSA (data3) for $\cos ^{2}(\pi x / 2)$ excitation, $N=1000, d_{\mathrm{av}}=2 \lambda$.

by the UA positioning. The beam pattern of the CSA is very close to that of the UA in near mainlobe. In far mainlobe, the averaged pattern is occurred by the RA. We can control the angle and the reduction level of the strong reduction of the SLL when we change the parameter $N$ in Eq. (11) as a variable number which is considered as fixed number in this section. In Sect. 4, we show a formula and some calculations by changing $N$ which corresponds to $c_{1}$ and $c_{2}$ in Eq. (13). The angle and the reduction level of the strong reduction of the SLL also depend on the element number $N$. It is very important to formulate the reduction of the SLL, however, we have not formulated it yet. By applying amplitude tapering with $\cos ^{2}(\pi x / 2)$ this level becomes a little higher. This effect may be attractive for MPT and does contribute for less area under the SLL.

From now on in the entire visible range will be used suitable amplitude excitation function which concerns the beamwidth and influence to transmitting efficiency and beam collection efficiency.

Let's see the behavior of our algorithm when $N$ increases and what could we expect as minimum of SLL. Now let's increase number of elements $N$ to 16000,32000 and 64000. In Fig. 7 and Fig. 8 we fix again entire visible range and no amplitude tapering use.

One can see the influence of number of elements over

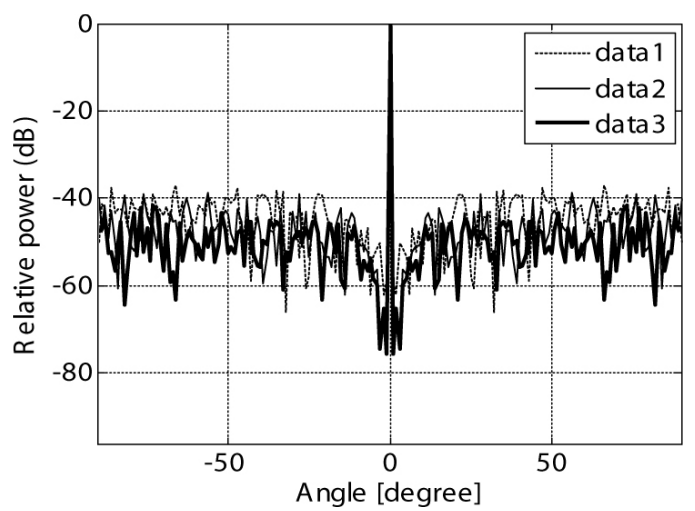

Fig. 7 Radiation characteristics of the CSA, $N=16000$ (data1), $N=32000$, (data2) and $N=64000$ (data3), $d_{\mathrm{av}}=2 \lambda$ and uniform excitation.

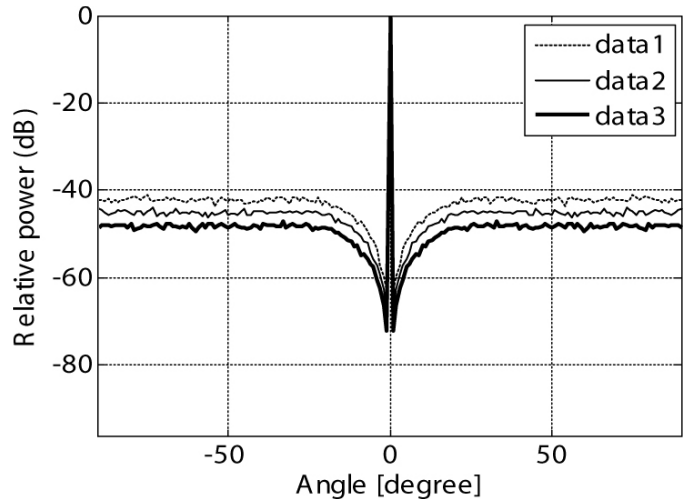

Fig. 8 Average patterns of the CSA algorithm with uniform excitation for $N=16000$ (data 1$), N=32000$ (data2) and $N=64000$ (data 3), $d_{\mathrm{av}}=2 \lambda$.

peak SLL. For $N=16000$ elements this level according to (5) is bellow $-35 \mathrm{~dB}$ with $90 \%$ probability of success. When increasing this number to $N=32000$ and 64000 the peak SLL is bellow $-37.5 \mathrm{~dB}$ and $-40 \mathrm{~dB}$ respectively. So a large amount of elements need to decrease a little peak SLL. The average SLL for $N=16000, N=32000$ and $N=64000$ is respectively $-42 \mathrm{~dB},-45 \mathrm{~dB}$ and $-48 \mathrm{~dB}-$ see Fig. 8 .

\section{Further Development of the Problem of Minimiza- tion of SLL}

Let's rewrite Eq. (11) as follows:

$$
\left\{x_{n}\right\}=c_{1}\left\{x_{n \operatorname{det}}\right\}+c_{2}\left\{x_{n \text { rand }} / N\right\}
$$

The influence of these two constants $c_{1}$ and $c_{2}$ over the behavior of the workspaces $\left\{x_{n \text { det }}\right\}$ and $\left\{x_{n \text { rand }}\right\}$ reveals an interesting properties of our algorithm, which was studied in previous section with $c_{1}=1$ and $c_{2}=1$. We have found experimentally the following set of constants.

$\left\{c_{1}=0.93, c_{2}=0.1\right\}$ to improve minimization of SLL and no gratings lobes appear-compare Fig. 9 and Fig. 7. Let call this model improved combined stochastic algorithm (ICSA). From now on into entire visible range will be used suitable amplitude excitation function which concerns the beamwidth and influence to transmitting efficiency and 


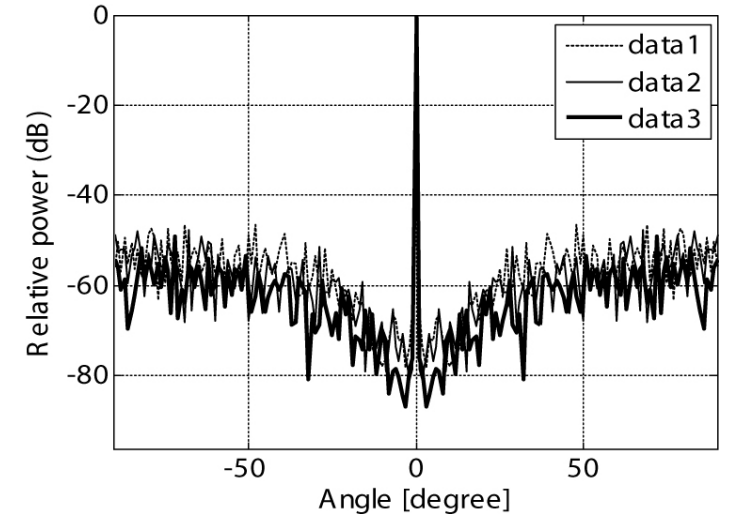

Fig. 9 Radiation characteristics of the ICSA, $N=16000$ (data1), $N=32000$, (data2) and $N=64000$ (data3), $d_{\mathrm{av}}=2 \lambda$ and $c_{1}=0.93, c_{2}=0.1$.

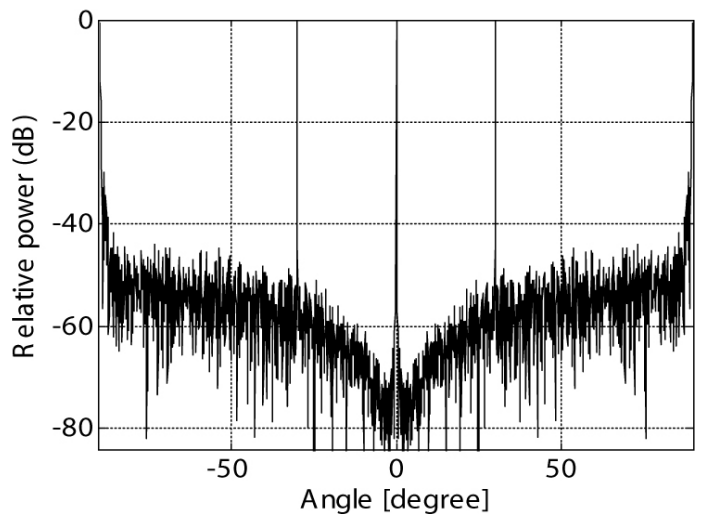

Fig. 10 Radiation characteristics of the ICSA, $N=16000, d_{\mathrm{av}}=2 \lambda, c_{1}=$ $1, c_{2}=0.1$.

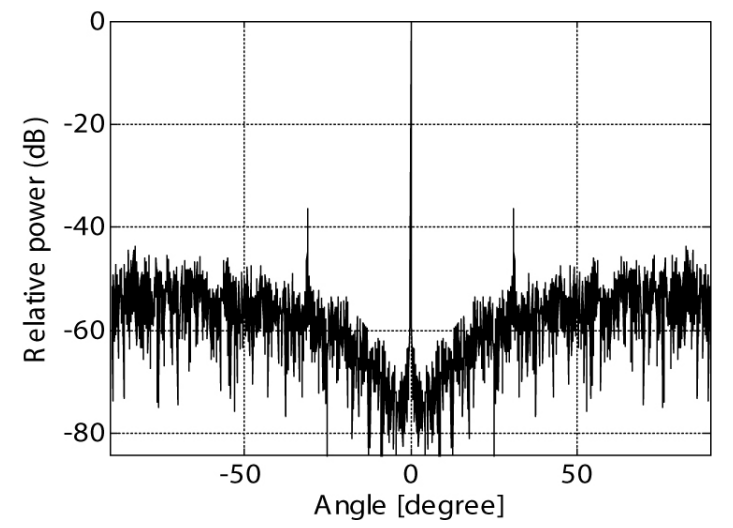

Fig. 11 Radiation characteristics of the ICSA, $N=16000, d_{\mathrm{av}}=2 \lambda, c_{1}=$ $0.97, c_{2}=0.1$.

beam collection efficiency.

Let study our algorithm with $c_{1}=1, c_{2}=0.1 ; c_{1}=$ $0.97, c_{2}=0.1 ; c_{1}=0.90, c_{2}=0.1$ for $N=16000$. To avoid false effect let enhance $N_{\text {data }}$ to 1801 - see Fig. 10 to Fig. 14. With not enough data point (for example $N=181$ ) the resolution is low and we can not register grating lobe. It is 'false effect.' One can see when decreasing $c_{2}$ grating lobes appear. Such grating lobes appear also for $N=32000$

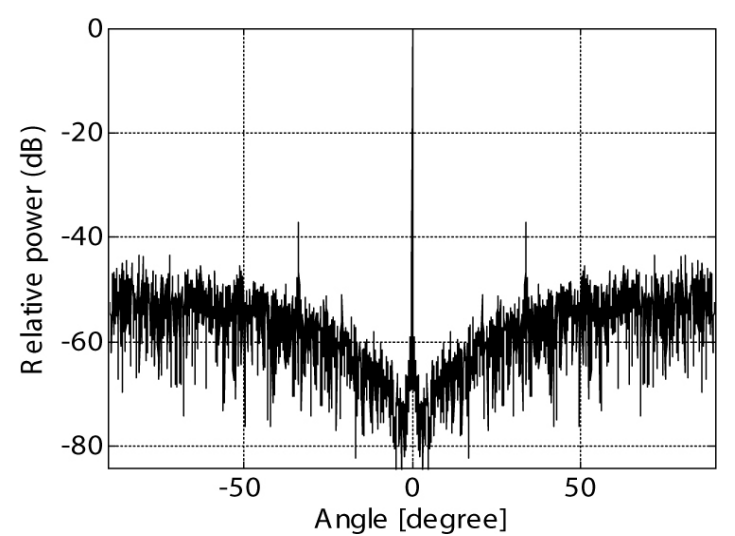

Fig. 12 Radiation characteristics of the ICSA, $N=16000, d_{\mathrm{av}}=2 \lambda, c_{1}=$ $0.90, c_{2}=0.1$

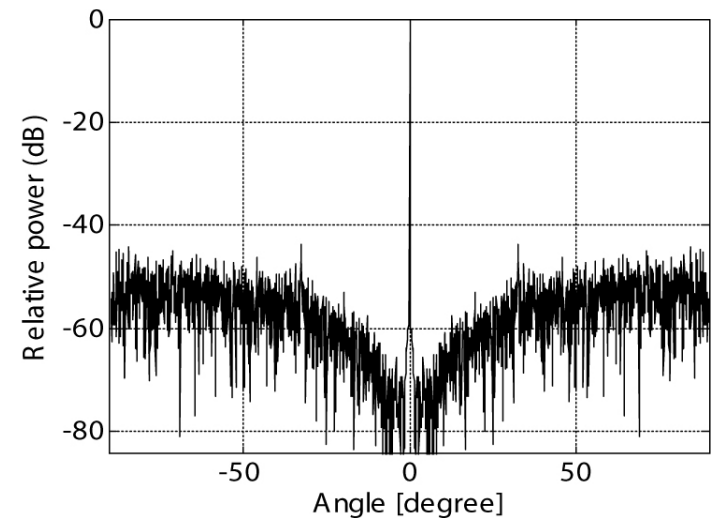

Fig. 13 Radiation characteristics of the ICSA, $N=16000, d_{\mathrm{av}}=2 \lambda, c_{1}=$ $0.93, c_{2}=0.1$

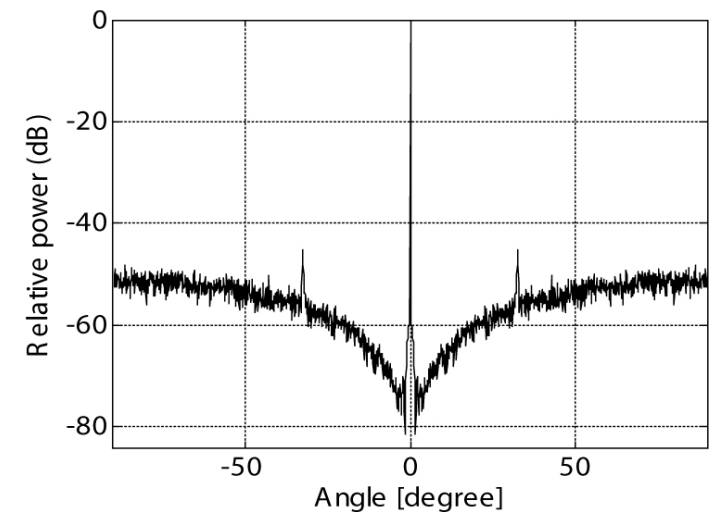

Fig. 14 Average patterns of the ICSA, $N=16000, d_{\mathrm{av}}=2 \lambda$ and $c_{1}=$ $0.93, c_{2}=0.1$.

and $N=64000$. We have found experimentally the optimum value of $c_{1}=0.93$. We have reduced SLL in comparison with Fig. 8 , independently that a little amount $(-45 \mathrm{~dB})$ of grating lobes remains. When decreasing $c_{2}$ further the SLL decreases too but grating lobes remains the same.

Let's further consider the opportunity of using our model with $d_{\mathrm{av}}=\lambda$. It is equivalent to shorten the aperture two times with the same number of elements. On the 


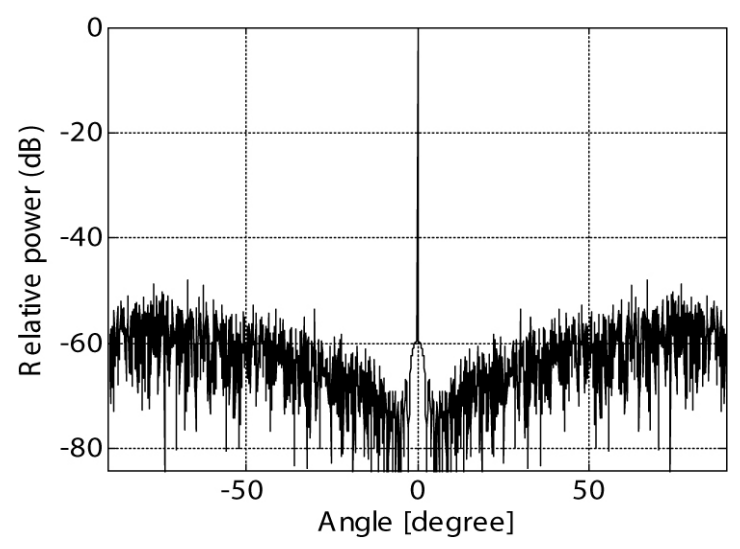

Fig. 15 Radiation characteristics of the ICSA, $N=16000, d_{\mathrm{av}}=\lambda, c_{1}=$ $0.93, c_{2}=0.1$

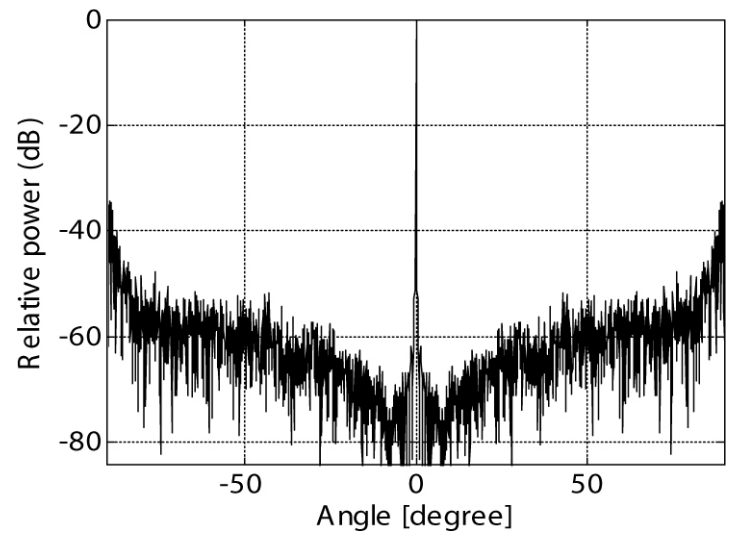

Fig. 16 Radiation characteristics of the ICSA, $N=16000, d_{\mathrm{av}}=\lambda, c_{1}=$ $0.99, c_{2}=0.1$.

Fig. 15 and Fig. 16 one can see Radiation characteristics and Average patterns for the set of constants $c_{1}=0.93, c_{2}=0.1$. At this we can increase $c_{1}$ up to 0.97 without grating lobes appear.

Comparing with Average patterns of Fig. 8 one can see a considerably improvement of SLL for $N=16000$ - from $-42 \mathrm{~dB}$ (In Lo's algorithm this average level is constant) to $-58 \mathrm{~dB}$. There is not grating lobes.

A little enhancement of $c_{1}$ to 0.99 causes again grating lobes - see Fig. 16.

Really, stochastic part of our algorithm plays the role of very small perturbation of deterministic workspace.

For $N=32000$ and $N=64000$ the aperture becomes two and four times larger, the beam becomes more and more narrow and transmitted power higher. However, for many reasons the aperture dimensions can not be limitless enlarged and this problem will be discussed further.

\section{Two-Dimensional Array}

Often, for a rectangular array aperture, a separable amplitude distribution is chosen and the array factors for UA (2) and RA (1) can be written as the product of two independent factors. For the RA with a rectangular aperture of $a b \lambda^{2}$

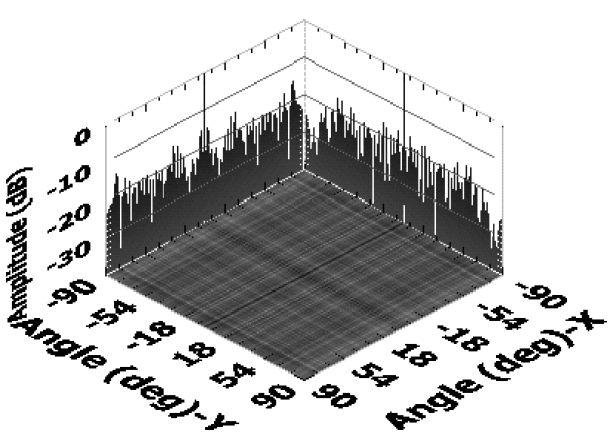

Fig. 17 Radiation characteristics of the RA with uniform excitation, $N=101$, average spacing, $d_{\mathrm{av}}=\lambda, \theta \in\left[-90^{\circ}, 90^{\circ}\right]$.

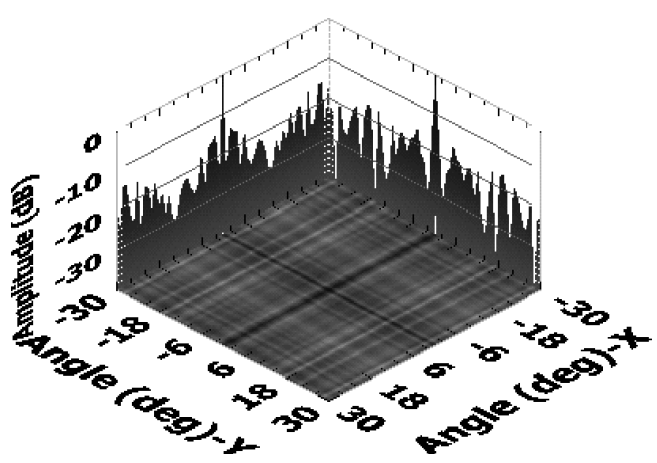

Fig. 18 Radiation characteristics of the RA with uniform excitation, $N=101$, average spacing, $d_{\mathrm{av}}=\lambda, \theta \in\left[-30^{\circ}, 30^{\circ}\right]$.

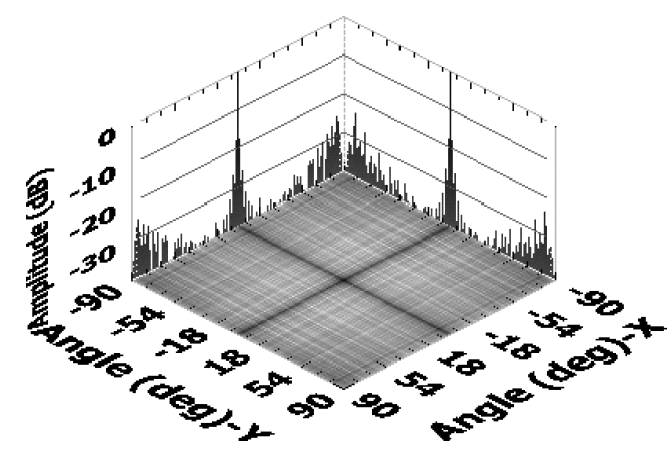

Fig. 19 Radiation characteristics of the ICSA with uniform excitation, $N=101$, average spacing, $d_{\mathrm{av}}=\lambda, \theta \in\left[-90^{\circ}, 90^{\circ}\right]$.

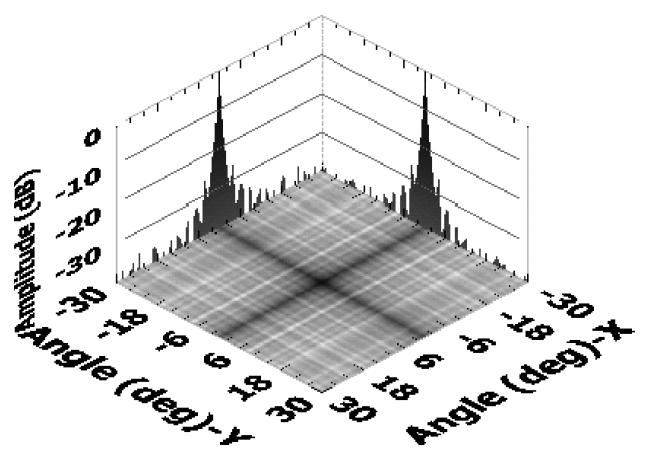

Fig. 20 Radiation characteristics of the ICSA with uniform excitation, $N=101$, average spacing, $d_{\mathrm{av}}=\lambda, \theta \in\left[-30^{\circ}, 30^{\circ}\right]$. 
in the $x y$ plane with a probability density function $f(x) f(y)$ is chosen. Then the relation between the total number of elements and the SLL is still approximately given by (5), except that $[4 a]$ is replaced by $[16 a b]$. The desired SLL will be achieved with a square of predetermined confident probability of success such as $0.9,0.95$ etc.

However, with elements 16000, 32000 and 64000 that we have used in our simulations it is impossible to simulate two-dimensional radiation patterns with ordinary computer resources. At Fig. 17 to Fig. 20 we have depicted twodimensional implementation of radiation characteristics of the algorithms RA and ICSA for $N=101$. One can see the same strong reduction of SLL occurs in the two-dimensional radiation patterns as in the one- dimensional radiation patterns.

\section{Transmitting and Beam Collection Efficiency}

To be determined the transmitting efficiency (TE) will be used the area under the peak SLL and the area under the main beam. Then transmitting efficiency can be presented as follows

$$
T E=\frac{M B_{\text {area }}}{M B_{\text {area }}+S L L_{\text {area }}}
$$

where $M B_{\text {area }}$ is main beam area and $S L L_{\text {area }}$ is side lobe area. The main beam area is defined as $4 \mathrm{~km} \phi$ at the center of beam in this paper.

When $N$ increases, the area of main beam decreases, but average SLL decreases too. It is not accurate to use average SLL, but rather the envelope of peak values of SLL obtained with large amount of observation points $N_{\text {data }}=$ 1801 in our simulations - see Fig. 15, which we have pre- pared also for $N=32000$ and $N=64000$. The envelop of peak values of SLL reaches respectively $-51 \mathrm{~dB},-53 \mathrm{~dB}$ and $-55 \mathrm{~dB}$.

In Fig. 21 we turn back again to the near zone which becomes narrower and narrower when the number of element $N$ increases. In this figure ICSA is with non-uniform

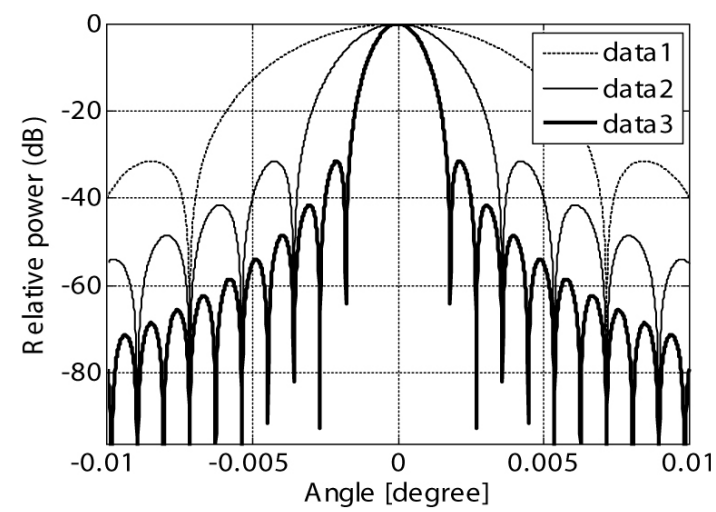

Fig. 21 Radiation characteristics of the ICSA average spacing $d_{\mathrm{av}}=\lambda$ with non-uniform excitation of $\cos ^{2}(\pi x / 2), N=16000$ (data1), $N=32000$ (data2) and $N=64000$ (data3).

Table 1 Characteristics of the transmitting part of the system for MPT.

\begin{tabular}{|c|c|c|c|c|c|}
\hline \multirow{2}{*}{$\begin{array}{l}\text { Number } \\
\text { of PCM }\end{array}$} & \multirow{2}{*}{\begin{tabular}{|c} 
Number of \\
Antenna \\
Elements \\
\end{tabular}} & \multirow{2}{*}{$\begin{array}{c}\text { Number of } \\
\text { elements in } \\
\text { Sub-array }\end{array}$} & \multirow{2}{*}{$\begin{array}{l}\text { Power } \\
{[\mathrm{GW}]}\end{array}$} & \multicolumn{2}{|c|}{$\begin{array}{r}\text { Diameter }[\mathrm{m}] \\
\mathrm{TE}\end{array}$} \\
\hline & & & & $\begin{array}{r}\mathrm{d}_{\mathrm{av}}=\mathrm{c}_{1} \lambda \\
\eta_{1 \mathrm{D}}\end{array}$ & $\eta_{2 \mathrm{D}}$ \\
\hline 1600 & 16000 & 10 & 1.02 & $\begin{array}{ll}769 & 0.9067\end{array}$ & 0.8221 \\
\hline 2134 & 32010 & 15 & 1.82 & $1538 \quad 0.8831$ & 0.7799 \\
\hline 2560 & 64000 & 25 & 2.62 & $3076 \quad 0.8580$ & 0.7362 \\
\hline
\end{tabular}
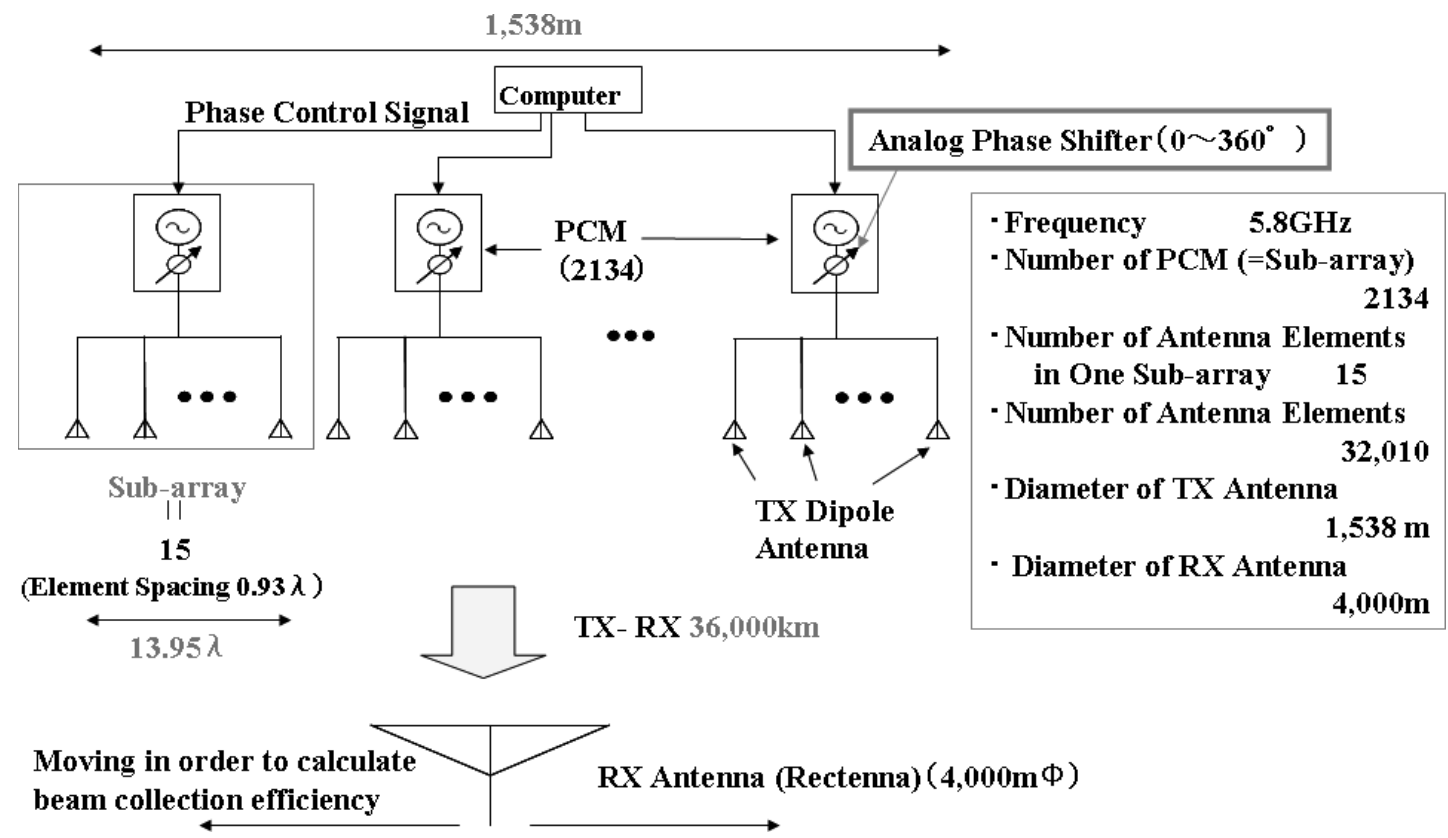

Fig. 22 Block chart of considerable SPS transmitting array. Parametrs are one example for calculation in Table 1. 
amplitude tapering with $\cos ^{2}(\pi x / 2)$.

Beam collection efficiency (BCE) of the MPT system is the ratio of power that is intercepted by the rectenna to the whole transmitted power and can be expressed as:

$$
B C E=\left.\int_{\theta_{r}}|P(\theta)|^{2} d \theta\left|\int_{\theta_{w}}\right| P(\theta)\right|^{2} d \theta
$$

where $|P(\theta)|^{2}$ is the power, $\theta_{r}$ is the angle sector due to rectenna and $\theta_{w}$ is the angle sector $\pm 90^{\circ}$. One can see from (15) that the lower SLL enhances the BCE.

In Table 1 are presented the features of the transmitting part of the system for MPT, which is shown in Fig. 22, depend on number of elements and possible reduction of workspace. A concept of "units" (sub-arrays or phased controlled magnetrons (PCM)) is adopted [26]. $\eta_{1 D}$ is one dimensional transmitting efficiency of linear array, calculated on the base of Eq. (14) and $\eta_{2 D}$ is two dimensional transmitting efficiency calculated as product of $\eta_{1 D}$ by itself and finally used; The diameter of transmitting antenna in [m] is indicated for different number of antenna elements.

The basic conclusion is that with ICSA we can achieve a low SLL and no grating lobes appear with reduced number of antenna elements in comparison with conventional Uniform array.

\section{Conclusion}

1. The pure stochastic algorithms with average spacing more than $\lambda$ (thinned arrays) really suppress grating lobes but on the price of sufficiently high SLL. So a large amount of elements need to decrease a little SLL.

2. Deterministic non-uniformly spaced algorithms are numerically difficult to implement for large antenna arrays. 3. We propose a new combined stochastic algorithm. It is a new unified approach in searching for reducing SLL by exploiting the interaction of deterministic and stochastic workspaces. It is a non-uniform spacing stochastic algorithm. Really, stochastic part of our algorithm plays the role of very small perturbations of deterministic workspace. So we have succeeded to reduce SLL considerably with out grating lobes appear and achieve high TE and BCE.

4. We propose a new concept of designing the transmitting part of the whole system for MPT.

5. Our study and simulation results clarify how to deal with the problems of side lobes and grating lobes in designing a large antenna array, which seems to be an important step toward the realization of future SPS/MPT systems.

\section{References}

[1] H. Matsumoto, "Research on solar power satellites and microwave power transmission in Japan," IEEE Microw. Mag., vol.3, no.4, pp.36-45, Dec. 2002.

[2] T.T. Taylor, "Design of line source antennas for narrow beamwidth and low sidelobes," IEEE Trans. Antennas Propag., vol.3, no.1, pp.16-28, Jan. 1955.

[3] R.E. Willey, "Space tapering of linear and planar arrays," IRE Trans AP, vol.10, pp.369-377, July 1962.
[4] H. Unz, "Linear arrays with arbitrarily distributed elements," IRE Trans. AP, vol.8, pp.222-223, March 1960.

[5] S.S. Sandler, "Some equivalences between equally and unequally spaced elements," IRE Trans. AP, vol.8, pp.496-500, Sept. 1960.

[6] R.F. Harrington, "Sidelobe reduction by nonuniform element spacing," IRE Trans. AP, vol.9, pp.187-192, March 1961.

[7] B.D. Steinberg, "Comparison between the peak sidelobe of the random array and algorithmically designed aperiodic arrays," IEEE Trans. Antennas Propag., vol.21, no.5, pp.366-369, May 1973.

[8] M.I. Skolnik, J.W. Sherman, III, and F.C. Ogg, Jr., "Statistically designed density-tapered arrays," IEEE Trans. Antennas Propag., vol.12, no.7, pp.408-417, July 1964.

[9] T. Numazaki, S. Mano, T. Kategi, and M. Mizusawa, "An improved thinning method for density tapering of planar array antennas," IEEE Trans. Antennas Propag., vol.35, no.9, pp.1066-1069, Sept. 1987.

[10] R.J. Mailloux and E. Cohen, "Statistically thinned arrays with quantized element weights," IEEE Trans. Antennas Propag., vol.39, no.4 pp.436-447, April 1991.

[11] T.M. Maher and D.K. Cheng, "Random removal of radiators from large linear arrays," IEEE Trans. Antennas Propag., vol.11, no.3, pp.106-112, March 1963.

[12] Y.T. Lo, "Sidelobe level in non-uniformly spaced antenna arrays," IEEE Trans. Antennas Propag., vol.11, no.7, pp.511-512, July 1963.

[13] Y.T. Lo, "A mathematical theory of antenna arrays with randomly spaced elements," IEEE Trans. Antennas Propag., vol.12, no.5, pp.257-268, May 1964.

[14] Y.T. Lo, "High resolution antenna arrays with elements at quantized random spacings," International Conference on Microwaves, Circuit Theory, and Information Theory, pp.379-380, Tokyo 1964.

[15] Y.T. Lo and S.W. Lee, "A study of space-tapered arrays," IEEE Trans. Antennas Propag., vol.14, no.1, pp.22-30, Jan. 1966.

[16] Y.T. Lo and R.J. Simcoe, "An experiment on antenna arrays with randomly spaced elements," IEEE Trans. Antennas Propag., vol.15, no.2, pp.231-235, March 1967.

[17] V.D. Agrawal and Y.T. Lo, "Mutual coupling in phased arrays of randomly spaced antennas," IEEE Trans. Antennas Propag., vol.20, no.3, pp.288-295, May 1972.

[18] B. Shishkov, H. Matsumoto, and N. Shinohara, "Probabilistic approach to design of large antenna arrays," Pliska Studia Mathemarica Bulgarica, vol.17, pp.249-269, 2005.

[19] B. Shishkov, H. Matsumoto, and N. Shinohara, "Mathematical modeling and optimization of the side lobe level of large antenna array," Proc. XXVIIIth G.A. of the URSI, New Delhi, India, HX.5(0265), Oct. 2005.

[20] B. Shishkov, N. Shinohra, H. Matsumoto, and K. Hashimoto, "On the minimization of sidelobes in large antenna arrays for microwave power transmission," Proc. 3rd International Symposium on Sustainable Energy System, pp.108-110, 2006.

[21] H. Matsumoto, N. Shinohara, and B. Shishkov, "Design method of array antenna instrument and its relevant instrument," Patent no.2003-368387, Oct. 29, 2003, (pending, Japan).

[22] B. Shishkov, N. Shinohra, K. Hashimoto, and H. Matsumoto, "On the optimization of sidelobes in large antenna arrays for microwave power transmission," IEICE Technical Report, SPS2006-11, 2006.

[23] R.J. Mailloux, Phased Array Antenna Handbook, Artech House, 2005.

[24] Ya.S. Shifrin, Statistical Antenna Theory, Golem, Boulder, CO, 1971

[25] H. Matsumoto, K. Hashimoto, and N. Shinohara, "Frequency problem for microwave power transmission (in Japanese)," Proc. 3rd SPS Symposium, pp.21-31, 2000.

[26] N. Shinohara, K. Nanokaichi, and H. Matsumoto, "Analysis of SPS microwave beam pattern in consideration of huge phase array structure (in Japanese)," Proc. 22nd ISAS Space Energy Symposium, pp.41-45, 2003. 


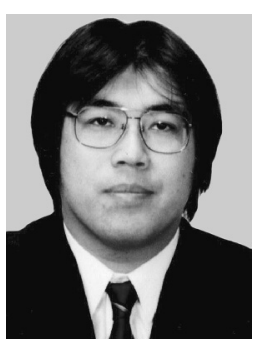

Naoki Shinohara received the B.E. degree in electronic engineering, the M.E. and Ph.D. (Eng.) degrees in electrical engineering from Kyoto University, Japan, in 1991, 1993 and 1998, respectively. He was a research associate in the Radio Atmospheric Science Center, Kyoto University from 1998. He was a research associate of the Radio Science Center for Space and Atmosphere, Kyoto University by recognizing the Radio Atmospheric Science Center from 2000, and there he was an associate professor since 2001. From 2004, he has been an associate professor in Research Institute for Sustainable Humanosphere, Kyoto University by recognizing the Radio Science Center for Space and Atmosphere. He has been engaged in research on Solar Power Station/Satellite and Microwave Power Transmission system. He is a member of the IEEE, URSI Commission C (Signals and Systems), and the IEEJ.

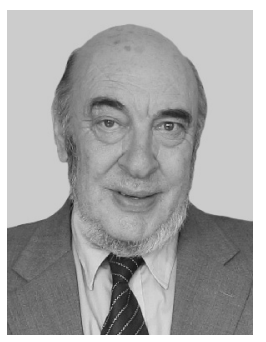

Blagovest Shishkov received the Ph.D. degree in Physics from the Institute of Electronics, Bulgarian Academy of Sciences and his D.Sc. degree from the Technical University of Sofia, Bulgaria, in 1974 and 1991 respectively. Presently, he is Head of Telecommunications Department in the Institute of Mathematics and Informatics at the Bulgarian Academy of Sciences. He has published more than a hundred research papers and three books in Signals Transmission (Signals, Filtering and Detection) and especially asymptotic methods in parameter estimation, signal detection and identification, pattern recognition and data quantization. His latest investigations and results are connected by signal processing of cyclostationary signals and detecting and studying of nonlinear wave interactions by using higher-order statistics. Both areas are successfully incorporated into adaptive antenna beamforming and analysis of time series associated with space data. The last investigation was devoted to mathematical modeling and optimization of the side lobes of large antenna arrays toward SPS/MPT applications. He was Visiting Professor in Japan (six times), France (three times), Spain etc. Official Member of the URSI Commission C (Signals and Systems), member of the IEEE, EURASIP, and IEICE (Japan).

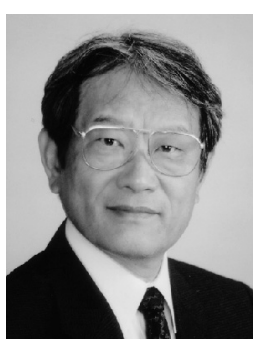

Hiroshi Matsumoto received the B.E. degree in electronic engineering, the M.E. and Ph.D. (Eng.) degrees in electrical engineering from Kyoto University, Japan, in 1965, 1967 and 1973, respectively. He was a professor of the Radio Atmospheric Science Center, Kyoto University from 1977, and there he was the director from 1992 to 1998 . He has been a professor in the Radio Science Center for Space and Atmosphere, Kyoto University by recognizing the Radio Atmospheric Science Center since 2000, and there he was the director from 2002 to 2004. From 2004 to 2005 , he was a professor and director in Research Institute for Sustainable $\mathrm{Hu}$ manosphere, Kyoto University by recognizing the Radio Science Center for Space and Atmosphere. From 2005, he has been an executive vice president of Kyoto University. He has been an advisory board in Communications Research Laboratory, Japan since 2001. He has been engaged in research on space plasma physics, space radio science and engineering, Solar Power Station/Satellite and Microwave Power Transmission system. He received the Tanaka Date Award from Society of Terrestrial Magnetism and Electricity of Japan in 1975, the NASA Group Achievement Award in 1993 and 1998, the American Geophysical Union (AGU) Fellow Award in 1999, the Shida-Rinzaburo Award in 1999 and the ISAP2000 Paper Award in 2000 and IEEE Fellow Award in 2003. He is an AGU Fellow, a member of Society of Geomagnetism and Earth, Planetary and Space Sciences (SGEPSS) and an IEEE Fellow. He was the president of the International Union of Radio Science (URSI) from 1999 to 2002.

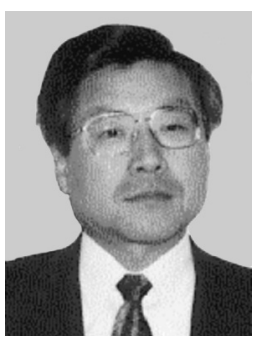

Kozo Hashimoto received the B.E., M.E and $\mathrm{Ph} . \mathrm{D}$. (Eng.) degrees in electrical engineering from Kyoto University, Japan, in 1969, 1971 and 1980, respectively. He was a research associate in the Faculty of Engineering, Kyoto University from 1974 to 1985 . He was an associate professor in the Faculty of Engineering, Tokyo Denki University from 1985 to 1989 , and there he was a professor from 1989 to 1995 . He was a professor in the Radio Atmospheric Science Center, Kyoto University from 1995, and a professor in the Radio Science Center for Space and Atmosphere (RASC), Kyoto University by reorganizing the Radio Atmospheric Science Center from 2000. Since 2004, he has been a professor in Research Institute for Sustainable Humanosphere, Kyoto University by reorganizing the RASC. He has been engaged in research on Solar Power Station/Satellite, Microwave Power Transmission system, wave phenomena in the Earth's magnetosphere plasma, and development of plasma wave analyzer. He received the Tanaka Date Award from Society of Terrestrial Magnetism and Electricity of Japan in 1984. He is a member of American Geophysical Union (AGU), a member of Society of Geomagnetism and Earth, Planetary and Space Sciences (SGEPSS) and a senior member of the IEEE. Geomagnetism and Earth, Planetary and Space Sciences (SGEPSS)and a senior member of the IEEE. 


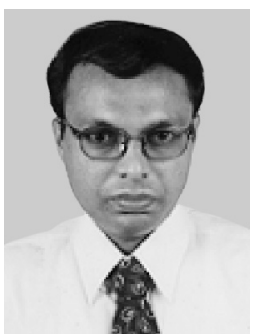

A.K.M. Baki received B.Sc. in Electrical and Electronic Engineering degree from Bangladesh University of Engineering and Technology (BUET), Bangladesh in 1992 and his M.Sc. degree from the University of Bolton (former Bolton Institute of Higher Education), UK in 2003 having followed a postgraduate program in Electronic System and Engineering Management at the South-Westphalia University of Applied Science, Germany. Currently he is working towards Ph.D. (Engg.) degree in electrical engineering in Kyoto University, Japan. From 2005 to 2006 he was a research assistant in the Research Institute for Sustainable Humanosphere (RISH), Kyoto University by recognizing the Radio Science Center for Space and Atmosphere (RASC). From 2004 to 2005 he was a research assistant in the Radio Science Center for Space and Atmosphere (RASC), Kyoto University. From 2003 to 2004 he was a research student in Radio Science Center for Space and Atmosphere (RASC), Kyoto University. From 2003 he has been a Sub-Divisional Engineer in Bangladesh Power Development Board (BPDB) from where he is now working on deputation towards his Ph.D. degree. From 1994 to 2003 he was an Assistant Engineer in BPDB. He is the former recipient of scholarship from German Academic Exchange Service (DAAD- Deutscher Akademischer Austauschdienst) for the study of his M.Sc. degree. He received Japan Government (Monbukagakusho: MEXT) scholarship for the study of his Ph.D. degree. He is a member of the IEEE. 